\title{
Influence of snail meat in the diet on mandibular bone loss in male rats: a densitometric, tomographic and morphometric study
}

\author{
MAREK BIEŃKO*, RADOSŁAW PIOTR RADZKI*, DARIUSZ WOLSKI*,**, PIOTR DĘBIAK ${ }^{* * *}$, \\ KRZYSZTOF SZKUCIK ${ }^{* * * *}$, MONIKA ZIOMEK ${ }^{* * * *}$, MICHAŁ GONDEK ${ }^{* * * *}$

\begin{abstract}
*Department of Animal Physiology, **Department of Animal Anatomy and Histology,
****Department of Food Hygiene of Animal Origin, Faculty of Veterinary Medicine, University of Life Sciences in Lublin, Akademicka 12, 20-950 Lublin, Poland

*** Laboratory of Radiology and Ultrasonography, Department and Clinic of Animal Surgery, Faculty of Veterinary Medicine, University of Life Sciences in Lublin, Głęboka 30, 20-612 Lublin, Poland
\end{abstract}

Bieńko M., Radzki R. P., Wolski D., Dębiak P., Szkucik K., Ziomek M., Gondek M. Influence of snail meat in the diet on mandibular bone loss in male rats: a densitometric, tomographic and morphometric study

\section{Summary}

The study was aimed at determining the effects of diets containing snail meat as the sole protein source, on mandible quality in male Wistar rats. In the experiment, three different snail-based diets were tested and compared with a casein-based control diet. These included snail meat from Helix pomatia, Cornu aspersum maxima and Cornu aspersum aspersum. In all diets, the protein content amounted to 10\% (as calculated on a dry weight basis). Forty male Wistar rats with an initial body mass of $50 \mathrm{~g} \pm 2$ were randomly allocated to one control and three experimental groups. After 28 days of experimental feeding, the rats were sacrificed. Their mandibles were isolated and investigated by densitometric (DXA), tomographic (pQCT) and morphometric methods. Moreover, the mechanical parameters (ultimate strength and Young's modulus) of the mandibles were measured. The results revealed that snail meat as the sole source of protein significantly decreased the bone mineral density (BMD) and content (BMC) of the mandibles. Moreover, the tomographic analysis demonstrated that each type of snail-based diet had a negative influence on the bone cortical and trabecular compartments (which was especially noticeable in the decreasing pQCT parameters). The investigation of mechanical resistance of the mandibles also revealed lower values of the ultimate strength and Young's modulus in the snail-based diet groups, compared with the casein group.

Keywords: mandibles, snail meat, pQCT, DXA, strength, rats

Many of the nutrients and food components consumed in our diets can have positive or negative influence on bone tissue development and health. Various mechanisms can be affected, including the modification of bone macro- and microstructure, the rate of bone metabolism, the functioning of the endocrine system, as well as the balance of mineral elements active in bone development (calcium, potassium and magnesium). Dietary factors, extending from minerals and vitamins to macronutrients (such as protein and fatty acids) and their proportions, derived from different types of diets (vegetarian, vegan, raw food or omnivorous), can influence bone health and condition, and thus also the risk of bone metabolic diseases, such as osteopenia or osteoporosis (22). Measurements of geometric, densitometric and mechanical properties are important in evaluating and describing bone tissue. Peripheral quantitative computed tomography (pQCT) has proven to be an effective tool in evaluating the densitometric and geometric properties of rat, mouse, and human bones, including oral bones $(4,8,12)$.

It is known that a high risk of tooth and oral bone loss is a consequence of postmenopausal status in women, who also show a systemic bone loss $(9,17,31)$. Tooth loss, alveolar bone resorption, and periodontal diseases may be associated with loss of mandibular bone 
mineral content (BMC). This situation is generally correlated with lumbar vertebral BMC, and therefore mandibular bone mass is strongly correlated with systemic bone mass $(19,30)$.

It should be remembered that oral health is one of the most important determinants of the quality of life. Therefore, people, both young and old, increasingly seek restorative dental treatment, including implant restoration, as well as periodontal and orthodontic treatment. All of these treatments are affected by the condition of the alveolar bone, which is the most important tissue supporting the teeth (11). Both prevention and, especially, a suitably balanced diet influence tooth quality. Animal protein includes all indispensable amino acids, and snail meat, in particular, is considered a high-quality food, rich in protein and iron, but low in fat. Hence, Adegoke et al. (1) suggest that diets containing snail meat may be important sources of protein for people requiring a high protein quality, low-fat diet. We advance the hypothesis that snail meat can affect the structure of the mandible during the growth and development of the body.

\section{Material and methods}

All animal procedures followed established guidelines for the care and handling of laboratory animals and were approved by the Local Animal Welfare Committee in Lublin, Poland (decision no. 70/2010). The study was carried out on 40 male Wistar rats with an initial body mass of $50 \mathrm{~g} \pm 2$. After 7 days of acclimatization, the rats were randomly divided into one control and three experimental groups (of 10 animals each). The rats were then housed individually with ad libitum access to food and water, being fed a standard diet (Agropol Motycz, Poland) with different sources of protein. In the control diet (CON group), the sole source of protein was casein, whereas in the experimental groups, protein originated from the meat of Helix pomatia (HP group), Cornu aspersum maxima (CAM group) or Cornu aspersum aspersum (CAA group). The content of protein in each diet, calculated on a dry weight basis, was constant and amounted to $10 \%$, as described before (27). The diet preparation procedure in this experiment was based on the methodology of the Association of Official Agricultural Chemists (23). After 28 days of experimental feeding, the rats were anaesthetized with carbon dioxide and euthanized by cervical dislocation. After euthanasia, their mandibles were isolated, cleaned of soft tissues, weighed and scanned by the dual X-ray absorptiometry (DXA) method to determine the bone mineral density (mBMD) and bone mineral content (mBMC) (4).

Densitometric analysis (DXA). The bone mineral density (mBMD) and bone mineral content (mBMC) of the mandibles were established with a Norland Excell Plus Densitometer (Fort Atkinson, WI, USA) equipped with Illuminatus DXA Software v.4.5, using the Small Animal Scan option. The measurements were performed with the following setting: scout scan speed $10 \mathrm{~mm} / \mathrm{s}$, resolution $0.5 \times 0.5 \mathrm{~mm}$; measurement scan speed $5 \mathrm{~mm} / \mathrm{s}$, resolution $0.5 \times 0.5 \mathrm{~mm}$. After a scout scan, the region of interest (ROI) was defined manually (4).

Peripheral quantitative computed tomography (pQCT). Right mandibles were scanned by peripheral quantitative computed tomography, using the XCT Research SA Plus system with software version 6.2 C (Stratec Medizintechnik GmbH, Pforzheim, Germany). The bones, placed in plastic tubes filled with $70 \%$ ethanol, were located centrally in the gantry of the tomograph and scanned in the centre of the mandibular first molar mesial root (Fig. 1). The region of interest for measurement was set by hand in such a way as to minimize the influence of the molar tooth (crown and root) and the incisor (root) (15). Using this procedure, total bone mineral content (Tot.BMC), total volumetric bone mineral density (Tot.vBMD), cortical compartment (as cortical volumetric bone density) (Ct.vBMD), cortical bone mineral content (Ct.BMC) and cortical thickness (Ct.Th) were assessed using peel mode 1 at a threshold value of $700 \mathrm{mg} / \mathrm{cm}^{3}$. Trabecular bone tissue density (Tb.vBMD), content (Tb.BMC) and area (Tb.Ar) were then measured using peel mode 2 at a threshold value of $390 \mathrm{mg} / \mathrm{cm}^{3}$. The initial scan (scout view) was performed at a speed of $10 \mathrm{~mm} / \mathrm{s}$, whereas the CT-scan (measurement scan) speed was $4 \mathrm{~mm} / \mathrm{s}(4)$.

Before every measurement series, both machines were calibrated according to set procedures with quality assurance phantoms (QA-Phantom) provided by the manufacturer.

Radiographic analysis. The mandibles were scanned with an X-Ray System apparatus model SRI SR-130 (Source-Ray, Inc. Ronkonkoma, NY, USA), using an FPD
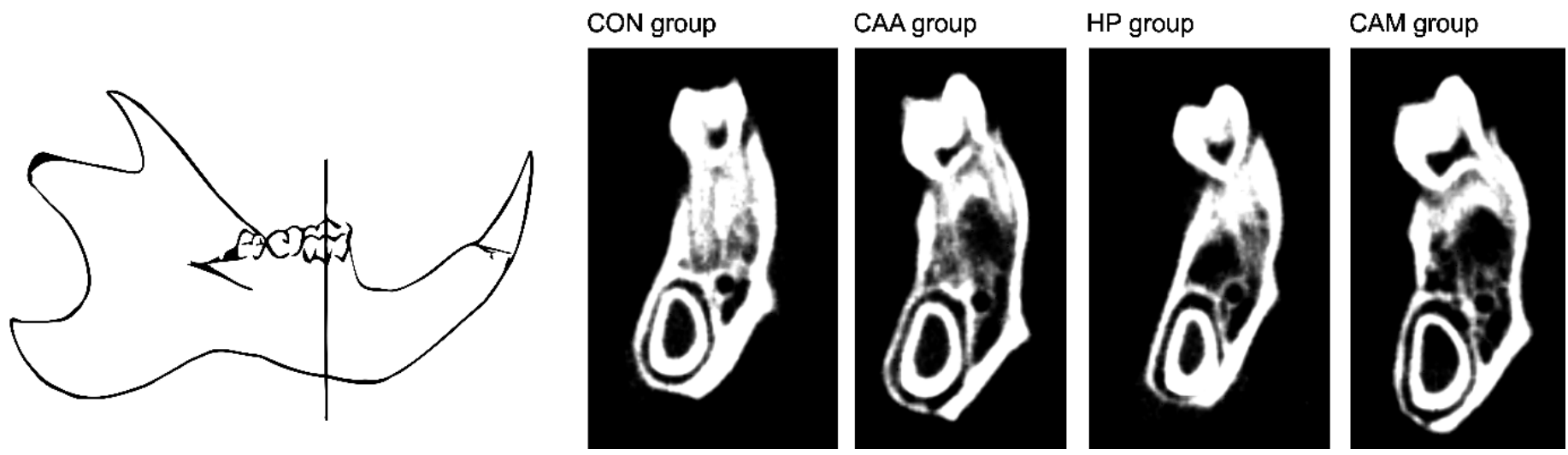

Fig. 1. Image of the rat mandible showing the measuring points in peripheral quantitative computed tomography (pQCT), and pQCT scans at the midpoint of the first molar in mandibles from the control (CON) and experimental (CAA, HP, CAM) groups 


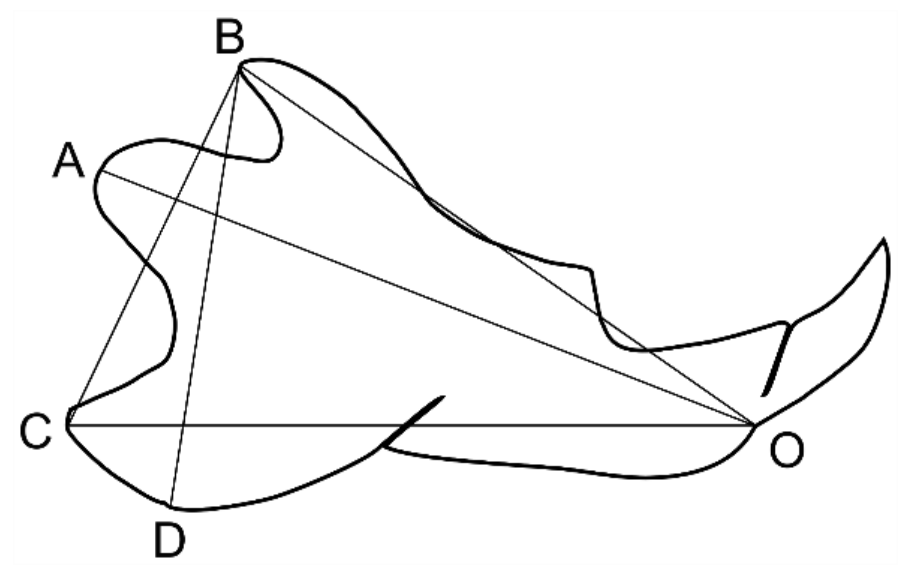

Fig. 2. Schematic image of the rat mandible (obtained by radiographic projection) showing the indices used to estimate mandibular size

Detector and the Leonardo DR System software. Radiograms for right and left parts of the mandible were made separately in the ML (medial-lateral) projection. The digital radiograms of the mandibles were measured manually with the use of the IRIS Software (Medi.com, Poland). Morphometric measurements were taken from these images according to current methodology, using stable points (anatomic landmarks) to obtain the parameters analyzed $(10,34)$. The following anatomic landmarks were identified (Fig. 2): the most anterior inferior point $(\mathrm{O})$, the superior posterior point of coronoid process (B), the superior posterior point of the condylar process (A), the inferior posterior point of the gonion $(\mathrm{C})$ and the inferior posterior point of the body (D).

Mandibular length (mm) was measured at the AO segment, mandibular height $(\mathrm{mm})$ at the BD segment, and mandibular base $(\mathrm{mm})$ at the $\mathrm{CO}$ segment. Mandibular area $\left(\mathrm{mm}^{2}\right)$ was calculated from the DXA measurement.

Three-point bending test. After DXA, pQCT and radiography measurements, the mandibles were placed on a ZwickRoell Z010 (ZwickRoell GmbH \& Co. KG, Ulm, Germany) universal testing machine with a $1 \mathrm{kN}$ measuring head (Xforce HP series). The measurements were performed with a span length of $10 \mathrm{~mm}$ at room temperature $(4,27)$. The samples were investigated as a flat model. In this experiment, the incisor present in the body of the mandible was not removed before testing, because its removal could fracture the mandible or alter its mechanical properties (15). We also hypothesized that the influence of the incisor on the densitometric, tomographic and morphometric properties of the mandible was the same for all groups. The mandibles were placed buccal side upwards, and the central loading point was aligned at the midpoint of the first molar (7). During the bending test, the loading force was compressed at a constant crosshead speed of $5 \mathrm{~mm} / \mathrm{min}$ until fracture. The data obtained were analyzed by the testXpert II 3.1 software, and the ultimate strength $\left(\mathrm{F}_{\text {max }}\right)$ and Young's modulus $\left(\mathrm{E}_{\text {mod }}\right)$ were subsequently determined $(4,27)$.

Statistical analysis. All results were reported as mean values $\pm \mathrm{SEM}$. A one-way analysis of variance (ANOVA) was used to test for significant differences between the groups. To detect significant differences between individual experimental groups, significant ANOVAs were followed by a post-hoc Tukey's test for multiple comparisons. Differences were considered significant at $p<0.05$. Analysis of significant differences was performed by the Statistica 13 software (StatSoft, Inc. Tulsa, TX, USA).

\section{Results and discussion}

The values of the bone mineral density and bone mineral content of the mandibles are presented in Table 1. The bone mineral content of the mandibles in the CAM, CAA and HP groups was lower (by $18.5 \%$, $12.3 \%$, and $11.6 \%$, respectively) than it was in the control group, and the differences were statistically

Tab. 1. Densitometric, tomographic and mechanical parameters of mandibles from the control (CON) and experimental (CAA, HP, CAM) groups

\begin{tabular}{|c|c|c|c|c|}
\hline Variables & CON group & CAA group & HP group & CAM group \\
\hline \multicolumn{5}{|c|}{ Densitometric analysis (DXA) } \\
\hline mBMD $\left(\mathrm{g} / \mathrm{cm}^{2}\right)$ & $0.098 \pm 0.0009$ & $0.096 \pm 0.0007$ & $0.094 \pm 0.0003$ & $0.093 \pm 0.0009 *, * * *$ \\
\hline mBMC (g) & $0.146 \pm 0.005$ & $0.128 \pm 0.005^{*}$ & $0.129 \pm 0.002^{*}$ & $0.119 \pm 0.003^{*}$ \\
\hline \multicolumn{5}{|c|}{ Tomographic analysis (pQCT) } \\
\hline Tot.BMC (mg/mm) & $7.84 \pm 0.53$ & $7.92 \pm 0.26$ & $7.02 \pm 0.62$ & $6.84 \pm 0.26$ \\
\hline Tot.vBMD $\left(\mathrm{mg} / \mathrm{mm}^{3}\right)$ & $720.81 \pm 15.44$ & $764.04 \pm 16.86$ & $709.66 \pm 12.65$ & $672.66 \pm 20.78^{* * *}$ \\
\hline Tb.BMC (mg/mm) & $1.79 \pm 0.18$ & $1.97 \pm 0.20$ & $1.28 \pm 0.11^{* *}$ & $1.28 \pm 0.15^{* * *}$ \\
\hline Tb.vBMD $\left(\mathrm{mg} / \mathrm{mm}^{3}\right)$ & $373.34 \pm 42.74$ & $424.21 \pm 43.94$ & $316.0 \pm 39.46$ & $290.45 \pm 46.20$ \\
\hline $\operatorname{Tb} . A r\left(m^{2}\right)$ & $4.89 \pm 0.29$ & $4.65 \pm 0.08$ & $4.49 \pm 0.40$ & $4.59 \pm 0.18$ \\
\hline Ct.BMC (mg/mm) & $6.69 \pm 0.24$ & $5.71 \pm 0.59$ & $3.81 \pm 0.34$ & $4.12 \pm 0.37$ \\
\hline Ct.vBMD (mg/mm³) & $1018.2 \pm 68.92$ & $939.75 \pm 51.02$ & $970.51 \pm 33.74$ & $924.81 \pm 40.64$ \\
\hline Ct.Th (mm) & $0.9162 \pm 0.17$ & $0.8455 \pm 0.18^{*}$ & $0.4683 \pm 0.09 * *$ & $0.5235 \pm 0.12^{* * * *}$ \\
\hline \multicolumn{5}{|c|}{ Three-point bending test } \\
\hline $\mathrm{F}_{\max }(\mathrm{N})$ & $55.17 \pm 2.09$ & $41.70 \pm 1.40^{*}$ & $40.89 \pm 1.23^{*}$ & $38.42 \pm 1.18^{*}$ \\
\hline $\mathrm{E}_{\text {mod }}(\mathrm{GPa})$ & $1.46 \pm 0.07$ & $1.06 \pm 0.13^{*}$ & $1.02 \pm 0.12$ & $1.19 \pm 0.11$ \\
\hline
\end{tabular}

Explanations: *vs. CON, **vs. CAA, ***vs. HP 
Tab. 2. Morphometric analysis of mandibles in the control (CON) and experimental (CAA, HP, CAM) groups

\begin{tabular}{|l|c|c|c|c|}
\hline \multicolumn{1}{|c|}{ Variables } & CON group & CAA group & HP group & CAM group \\
\hline Length $(\mathrm{mm})$ & $23.76 \pm 0.23$ & $23.03 \pm 0.22$ & $23.26 \pm 0.19$ & $22.17 \pm 0.29^{*}, * *, * *$ \\
\hline Base $(\mathrm{mm})$ & $22.10 \pm 0.31$ & $21.58 \pm 0.20$ & $21.33 \pm 0.15$ & $20.41 \pm 0.23^{*, * * * * *}$ \\
\hline Height $(\mathrm{mm})$ & $12.35 \pm 0.21$ & $11.89 \pm 0.14^{*}$ & $11.69 \pm 0.11$ & $12.06 \pm 0.14$ \\
Area $\left(\mathrm{mm}^{2}\right)$ & $1.49 \pm 0.05$ & $1.33 \pm 0.02^{*}$ & $1.36 \pm 0.02^{*}$ & $1.28 \pm 0.033^{*}$ \\
\hline Mandible mass $(\mathrm{mg})$ & $348.7 \pm 0.009$ & $321.9 \pm 0.01^{*}$ & $310.6 \pm 0.006$ & $275.9 \pm 0.007^{*}$ \\
\hline
\end{tabular}

Explanations: as in Tab. 1

significant $(\mathrm{p}<0.05)$. The same tendency was observed in relation to the bone mineral density.

The results of the pQCT analysis of cortical and trabecular bone tissue of the mandibles are shown in Table 1 . The analyses of the cortical compartment by pQCT techniques revealed that the values of cortical bone mineral content (Ct.BMC), cortical volumetric bone mineral density (Ct.vBMD) and cortical thickness (Ct.Th) were lower in all experimental groups, compared with the control, but only the differences in cortical thickness were statistically significant $(p<0.05)$. The analysis of trabecular bone found a similar tendency, but only for the CAM and HP groups. In the CAA group, the values of total bone mineral content (Tot.BMC), total volumetric bone mineral density (Tot.vBMD), trabecular bone mineral content (Tb. BMC) and trabecular volumetric bone mineral density (Tb.vBMD) were higher than they were in the control group (by $1.02 \%, 5.9 \%, 10 \%$ and $13.6 \%$, respectively).

In the CAM group, the experimental diet had a negative and statistically significant influence on mandible length and base (Tab. 2). In the remaining groups, these parameters were at a similar level as in the control. The area of the mandibles in the CAM, HP and CAA groups (as measured by DXA) was also lower (by 14\%, 8.7\% and $10.7 \%$, respectively) than it was in the control group, and the differences were statistically significant $(\mathrm{p}<0.05)$ (Tab. 2).

The experimental diet had a negative influence on the ultimate strength of the mandibles analyzed. The value of this parameter in the CAM, HP and CAA groups was lower (by $30.4 \%, 25.8 \%$ and $24.4 \%$, respectively) than it was in the control group, and the difference was statistically significant $(\mathrm{p}<0.05)$. The experimental diet also had a negative, but statistically insignificant, effect on the elasticity modulus (Tab. 1).

The results of mandible mass measurements are presented in Table 2. It can be seen that all experimental diets decreased this parameter, but statistically significant $(p<0.05)$ differences were observed only in the CAM group.

This study compared bone examination results in rats receiving a standard diet based on casein and three types of diet based on the most frequently consumed species of snails: Helix pomatia, Cornu aspersum maxima and Cornu aspersum aspersum. Mollusc meat is considered to be a nutritious food, providing the consumer with several essential amino acids and proteins, as well as several vitamins and minerals (1, 33). Humans have been consuming snails since ancient times, and today snails (fresh or frozen) are a common food item for people worldwide. The most preferred and consumed snail species are Helix aspersa (the garden snail), commonly found in western Europe and the northern Mediterranean areas, and Helix pomatia (Roman or edible snail), which occurs in central and south-eastern Europe. Garden snail meat has similar contents of proteins, amino acids, vitamins, minerals and fatty acid as many kinds of seafood $(5,35)$. Indeed, its mineral content (calcium, potassium, phosphorus and sodium) is comparable with that of the Atlantic bonito (5). All of these nutrients play important roles in human metabolic processes and immune system activity. Cagiltay et al. (5) found that $100 \mathrm{~g}$ of Helix aspersa meat contained $5.46 \mathrm{mg}$ of vitamin A, $0.88 \mathrm{mg}$ of vitamin $\mathrm{E}, 0.154 \mathrm{mg}$ of vitamin $\mathrm{B}_{1}, 0.065 \mathrm{mg}$ of vitamin $B_{2}, 3.22 \mathrm{mg}$ of vitamin $B_{3}$ and $0.28 \mathrm{mg}$ of vitamin $\mathrm{B}_{6}$. For the sake of comparison, the daily requirement of a healthy person is $1.5 \mathrm{mg}$ of vitamin $\mathrm{A}, 17 \mathrm{mg}$ of vitamin $\mathrm{E}, 1.4 \mathrm{mg}$ of vitamin $\mathrm{B}_{1}, 1.6 \mathrm{mg}$ of vitamin $B_{2}, 17 \mathrm{mg}$ of vitamin $B_{3}$ and $1.9 \mathrm{mg}$ of vitamin $\mathrm{B}_{6}$ (3). Snail meat is also rich in high-qulity protein, but low in fat. Thus, it can be an alternative nutriment for people who require low-fat diets containing highquality protein (5).

Although the nutritional composition of edible snails has been known for years, there is practically only one publication on the influence of a "snail diet" on bone health and condition (27). The present study is the first investigation of the influence of a diet consisting mostly of snail-derived protein on the quality and functional status of the mandible.

The mandible is composed of two kinds of bone tissue: cortical tissue and trabecular tissue. The trabecular one is more active in remodelling processes, but less mineralized. Bone diseases are primarily related to disorders in the remodelling balance, which is maintained by osteoclast and osteoblast activity, calcium-regulating factors, growth factors, cytokines, sex hormones and other factors, such as diet, drugs, stimulants or the environment (6).

Dual X-ray absorptiometry (DXA) and peripheral quantitative tomography (pQCT) are the criterion standard for the evaluation and diagnosis of bone condition, 
They are very sensitive and reproducible techniques for measuring bone quality, not only in humans, but also in small animals (4, 25-28).

The quality of bone tissue is also measured by its resistance to fractures which occur when loads exceed the bone strength. Trabecular thickness, measured by pQCT, correlates with stiffness, while cortical thickness correlates with the breaking force (2). Furthermore, the mechanical competence of cortical bone tissue depends not only on BMD, but also on the structural properties of the cortical compartment, such as peripheral circumference and cortical area. Many factors, including the type of diet, may affect bone tissue quality and bring about an increase or decrease in bone mineral density and bone mineral content (22).

The results of this experiment, based on DXA measurements, distinctly demonstrated that snail meatbased diets significantly decreased the bone mineral content of mandibles. What is more, the values of bone mineral density were lower in the groups receiving a sole snail diet, especially in the CAM group, than they were in the control group. Both trabecular and cortical compartments of bone tissue are very sensitive to any influences affecting bone metabolism. However, mostly the changes in trabecular bone tissue are more intensive $(25,28)$. In our study, the tomographic analysis demonstrated a negative influence of each type of snail-based diet on both compartments of bone (cortical and trabecular). This was especially noticeable in decreasing pQCT parameters, such as Tb.BMC, Tb.vBMD, Tb.Ar, Ct.BMC and Ct.vBMD. In addition, the investigation of the mechanical resistance of the mandibles revealed lower values of the ultimate strength and Young's modulus in the snail-meat diet groups, compared with the casein group control. It can be said, then, that such snail-based diets result in a deterioration of bone tissue and increase its susceptibility to structural failures, such as stress cracking or fracture.

It is important to determine what happened to the mandibles after the consumption of the snail meatbased diets. Were the abovementioned effects due to metabolic bone disorders or to grow retardation? It is possible that the consumption of snail meat as the sole source of protein causes effects similar to osteopenia or osteoporosis. The changes in the structure of the mandibles and their mechanical properties also resembled those in gonadectomized animals $(24-26,28)$. In the last 20 years, a number of publications have demonstrated that the systemic bone mass loss that causes osteoporosis is highly correlated with the loss of teeth. These studies (among others) suggest that the loss of mandibular bone mineral content in postmenopausal women is correlated with the lumbar vertebral bone mineral content, and that mandibular bone mass is strongly correlated with systemic bone mass (7, 16-19, $31)$. For example, Jiang et al. $(13,14)$ demonstrated that dietary calcium deficiency and ovariectomy in rats induced significant decreases in trabecular and cortical bone mineral density and content in the mandible, while Lerouxel et al. (21) reported that sex hormone deprivation induced alveolar bone loss in the male rat. In these studies, as in ours, the mandibular bone loss was evidenced with BMD/BMC parameters measured by dual energy absorptiometry. Similar effects were demonstrated by Tanaka et al. (32) and Kuroda et al. (20) in post-ovariectomy rats one year after the procedure.

The results of our experiment contradict the findings of Sarkar et al. (29). They reported that flesh extracts from the water snail (Viviparous bengalensis) significantly inhibited the development and progression of experimental osteoporosis in bilaterally ovariectomized Wistar rats and of osteoarthritis induced in male Wistar rats by bacterial collagenase injection, but they based their hypothesis on the biochemical analysis of urine and blood serum without bone tissue investigation.

In conclusion, the consumption of snail meat as the sole source of protein had negative influence on the metabolism of mandibles in male rats, making them fragile and reducing their density. However, further investigations are necessary to provide more information on the mechanism by which snail meat affects general growth and development, especially with regard to mandibles.

\section{References}

1. Adegoke A. A., Bukola A. C., Comfort I. U., Olayinka A. A., Amos K. O.: Snails as meat source: Epidemiological and nutritional perspectives. J. Microbiol. Antimicrob. 2010, 2, 1-5.

2. Beaupied H., Lespessailles E., Benhamou C. L.: Evaluation of macrostructural bone biomechanics. Joint Bone Spine 2007, 74, 233-239.

3. Belitz H. D., Grosch W., Schieberle P.: Das Lehrbuch der Lebensmittelchemie Springer-Verlag, Berlin Heidelberg 2008.

4. Bieńko M., Radzki R. P., Wolski D.: The peripheral quantitative computed tomographic and densitometric analysis of skeletal tissue in male Wistar rats after chromium sulfate treatment. Annals Agric. Environ. Med. 2017, 24 446-452.

5. Cagiltay F., Erkan N., Selçuk A.: Amino acid, fatty acid, vitamin and mineral contents of the edible garden snail (Helix aspersa). J. Fish. Sci. 2011, 5, 354-363.

6. Chapurlat R. D.,Confavreux C. B.: Novel biological markers of bone: from bone metabolism to bone physiology. Rheumatology, Oxford 2016, 55, 1714-1725.

7. Elovic R. P., Hipp J. A., Hayes W. C.: Ovariectomy decreases the bone area fraction of the rat mandible. Calcif. Tissue Int. 1995, 56, 305-310.

8. Ferretti J. L., Capozza R. F., Zanchetta J. R.: Mechanical validation of a tomographic (pQCT) index for noninvasive estimation of rat femur bending strength. Bone 1996, 18, 97-102.

9. Gilles J. A., Carnes D. L., Dallas M. R., Holt S. C., Bonewald L. F.: Oral bone loss is increased in ovariectomized rats. J. Endod. 1997, 23, 419-422.

10. Guerreiro F. S., Diniz P., Carvalho P. E., Ferreira E. C., Avancini S. R., Ferreira-Santos $R$. I.: Effects of masticatory hypofunction on mandibular morphology, mineral density and basal bone area. Braz. J. Oral Sci. 2013, 12, 205-211.

11. Howson C. P.: Perspectives and needs for health in the $21^{\text {st }}$ century: $20^{\text {th }}$-century paradigms in $21^{\text {st }}$-century science. J. Hum. Virol. 2000, 3, 94-103.

12. Jamsa T., Jalovaara P., Peng Z., Vaananen H. K., Tuukkanen J.: Comparison of three-point bending test and peripheral quantitative computed tomography analysis in the evaluation of the strength of mouse femur and tibia. Bone 1998, $23,155-161$ 
13. Jiang G., Matsumoto H., Fujii A.: Mandible bone loss in osteoporosis rats J. Bone Miner. Metab. 2003, 21, 6, 388-395.

14.Jiang G., Matsumoto H., Yamane J., Kuboyama N., Akimoto Y., Fujii A. Prevention of trabecular bone loss in the mandible of ovariectomized rats. J. Oral Sci. 2004, 46, 75-85.

15.Jiang G. Z., Matsumoto H., Hori M., Gunji A., Hakozaki K., Akimoto Y., Fujii A.: Correlation among geometric, densitometric, and mechanical properties in mandible and femur of osteoporotic rats. J. Bone Miner. Metab. 2008, 26, 130-137.

16. Jonasson G., Kiliaridis S., Gunnarsson R.: Cervical thickness of the mandibular alveolar process and skeletal bone mineral density. Acta Odontol. Scand. 1999 57, 155-161

17. Krall E. A., Garcia R. I., Dawson-Hughes B.: Increased risk of tooth loss is related to bone loss at the whole body, hip, and spine. Calcif. Tissue Int. 1996, 59, 433-437.

18. Kribbs P. J.: Comparison of mandibular bone in normal and osteoporotic women. J. Prosthet. Dent. 1990, 63, 218-222.

19. Kribbs P. J., Chesnut C. H. III, Ott S. M., Kilcoyne R. F.: Relationships between mandibular and skeletal bone in a population of normal women. J. Prosthet. Dent. 1990, 63, 86-89.

20. Kuroda S., Mukohyama H., Kondo H., Aoki K., Ohya K., Ohyama T., Kasugai S. Bone mineral density of the mandible in ovariectomized rats: analyses using dual energy X-ray absorptiometry and peripheral quantitative computed tomography. Oral Dis. 2003, 9, 24-28.

21. Lerouxel E., Libouban H., Moreau M. F., Basle M. F., Audran M., Chappard D. Mandibular bone loss in an animal model of male osteoporosis (orchidectomized rat): a radiographic and densitometric study. Osteoporos. Int. 2004, 15, 814-819.

22. Palacios C.: The role of nutrients in bone health, from A to Z. Crit. Rev. Food Sci. Nutr. 2006, 46, 621-628.

23. Paszkiewicz W., Ziomek M., Szkucik K., Mackowiak-Dryka M.: Production and quality of snail meat. Vet. Med. Sci. Pract. 2014, 70, 673-679.

24. Radzki R. P., Bieńko M., Filip R., Albera E., Kankofer M.: Effect of Strontium Ranelate on Femur Densitometry and Antioxidative/Oxidative Status in Castrated Male Rats. Scand. J. Lab. Anim. Sci. 2009, 36, 193-201.
25.Radzki R. P., Bieńko M., Filip R., Pierzynowski S. G.: The Protective and Therapeutic Effect of Exclusive and Combined Treatment with Alphaketoglutarate Sodium Salt and Ipriflavone on Bone Loss in Orchidectomized Rats. J. Nutr. Health Aging 2016, 20, 628-636.

26. Radzki R. P., Bieńko M., Pierzynowski S. G.: Anti-osteopenic effect of alphaketoglutarate sodium salt in ovariectomized rats. J. Bone Miner. Metab. 2012, 30, 651-659.

27. Radzki R. P., Bieńko M., Polak P., Szkucik K., Ziomek M., Ostapiuk M., Bienias $J$. : Is the consumption of snail meat actually healthy? An analysis of the osteotropic influence of snail meat as a sole source of protein in growing rats. J. Anim. Physiol. Anim. Nutr. 2017, DOI: 10.1111/jpn.12851.

28. Radzki R. P., Bieńko M., Wolski D., Lis A., Radzka A.: Lipoic acid stimulates bone formation in ovariectomized rats in a dose-dependent manner. Can. J. Physiol. Pharmacol. 2016, 94, 947-954.

29. Sarkar A., Datta P., Gomes A., Gupta S., Gomes A.: Anti-Osteoporosis and Anti-Osteoarthritis Activity of Fresh Water Snail (Viviparous bengalensis) Flesh Extract in Experimental Animal Model. Open J. Rheumatol. Autoimmune Dis. 2013, 3, 10-17.

30. Taguchi A., Tanimoto K., Suei Y., Ohama K., Wada T.: Relationship between the mandibular and lumbar vertebral bone mineral density at different postmenopausal stages. Dentomaxillofac. Radiol. 1996, 25, 130-135.

31. Taguchi A., Tanimoto K., Suei Y., Wada T.: Tooth loss and mandibular osteopenia. Oral Surg. Oral Med. Oral Pathol. Oral Radiol. Endod. 1995, $79,127-132$.

32. Tanaka M., Toyooka E., Kohno S., Ozawa H., Ejiri S.: Long-term changes in trabecular structure of aged rat alveolar bone after ovariectomy. Oral Surg. Oral Med. Oral Pathol. Oral Radiol. Endod. 2003, 95, 495-502.

33. Thanonkaew A., Benjakul S., Visessanguan W.: Chemical composition and thermal property of cuttlefish (Sepia pharaonis) muscle. J. Food Comp. Anal. 2006, 19, 127-133.

34. Ubios A. M., Piloni M. J., Cabrini R. L.: Mandibular growth and tooth eruption after localized x-radiation. J. Oral Maxillofac. Surg. 1992, 50, 153-156.

35. Udoh A. P., Akpanyung E. O., Igiran I. E.: Nutrients and anti-nutrients in small snails (Limicolaria aurora). Food Chemistry 1995, 53, 239-241.

Corresponding author: Marek Bieńko D.Sc., PhD, Akademicka 12, 20-950 Lublin, Poland; e-mail: marek.bienko@up.lublin.pl 